\title{
F3-targeted cisplatin-hydrogel nanoparticles as an effective therapeutic that targets both murine and human ovarian tumor endothelial cells in vivo
}

\author{
I. Winer ${ }^{1}$, S. Wang ${ }^{2}$, Y-E K. Lee ${ }^{2}$, W. Fan ${ }^{2}$, Y. Gong ${ }^{3}$, D. Burgos-Ojeda ${ }^{3}$, G. Spahlinger ${ }^{3}$, R. \\ Kopelman', and Ronald J. Buckanovich, MD, $\mathrm{PhD}^{1,3}$ \\ ${ }^{1}$ Division of Gynecologic Oncology, Department of Obstetrics and Gynecology, University of \\ Michigan Medical Center, Ann Arbor, Ml 48109 \\ 2 Department of Chemistry, University of Michigan Medical Center, Ann Arbor, MI 48109 \\ ${ }^{3}$ Division of Hematology Oncology, Department of Internal Medicine, University of Michigan Medical \\ Center, Ann Arbor, MI 48109
}

\begin{abstract}
Recent studies indicate that ovarian cancer may be highly responsive to anti-vascular therapeutics. We have developed an anti-vascular tumor therapeutic using the $\mathrm{F} 3$ peptide to target cisplatin loaded nanoparticles (F3-Cis-Np) to tumor vessels. We demonstrate that while F3-Cis-Np bind with high specificity to both human ovarian tumor cells and tumor endothelial cells in vitro, they only demonstrate cytotoxic activity against the tumor endothelial cells. In vivo these nanoparticles bind primarily to tumor endothelial cells. Therapeutic studies in both flank and orthotopic intraperitoneal murine ovarian tumor models, as well as human tumor xenograft models, demonstrate rapid tumor regression with treatment. Treatment was associated with significant vascular necrosis consistent with an anti-vascular effect. Furthermore treatment was active in both platinum sensitive and platinum resistant cell lines. Importantly we demonstrate that F3-Cis-Np bind to human tumor endothelial cells in vitro and to human tumor vessels in vivo. Therapy targeting human vasculature in vivo with F3-Cis-Np led to near complete loss of all human tumor vessels in a murine model of human tumor vasculature. Our studies indicate that F3-targeted vascular therapeutics may be an effective treatment modality in human ovarian cancer.
\end{abstract}

\section{Keywords}

F3-peptide; nanoparticles; ovarian cancer; therapy; tumor vessels

\section{Introduction}

Ovarian cancer is a deadly disease for which there have been few new therapies. For a decade platinum and taxane chemotherapy regimens remain the mainstay of therapy. Recent studies have suggested angiogenic pathways are important therapeutic targets in ovarian cancer. Phase II trials suggest a significant response rate of ovarian cancer to anti-VEGF therapy (1-4).

This is unlike other solid tumors such as colon, lung, and breast cancer which showed no response to single agent anti-VEGF therapy. When anti-VEGF therapy is used in combination 
with chemotherapy in ovarian cancer, response rates are even higher $(5,6)$. Unfortunately, even when used in combination with chemotherapy, response to anti-VEGF therapy is relatively short. This is due in part to host cell up-regulation of alternate angiogenic pathways (7).

Targeting vascular cells directly represents a means to target numerous angiogenic pathways which act ultimately at the endothelial cell. In addition, unlike traditional anti-angiogenic therapies which prevent new vasculature, and therefore typically lead to disease stabilization but not regression, targeting active tumor vascular cells could potentially lead to tumor necrosis and disease regression. Ovarian tumor vasculature has been demonstrated to be unique from normal resting vasculature $(8,9)$. Several peptides have been identified and developed which bind with relatively high affinity and specificity to tumor vessels. RGD (arginine-glycineaspartic acid) motif containing peptides have been developed which bind to integrin molecules which are up-regulated on tumor vessels and sometimes tumor cells $(10,11)$. Similarly, aspargine-glycine-arginine peptides can be used to target CD13 isoforms expressed in tumor vasculature (12). The 31 amino acid F3 peptide has also been shown to bind to nucleolin protein expressed on the surface of tumor endothelial cells as well as on the surface tumor cells (13$15)$.

These peptides have been used to target nanoparticles carrying various therapeutic payloads to the tumor, such as MRI contrast agents and photodynamic drugs $(16,17)$. This approach allows the therapeutic agent to be concentrated at the tumor site, while reducing systemic exposure and potentially reducing drug related side effects. RGD targeted cisplatin nanoparticles were found to inhibit endothelial cell proliferation in vitro (18). RGD targeted paclitaxel containing nanoparticles were demonstrated to target ovarian cancer cells in vitro and in vivo and effectively restrict ovarian tumor growth (19). Similarly, in several tumor models including ovarian cancer, vascular targeted liposomal doxorubicin was found to be a more effective therapeutic than traditional doxorubicin or liposomal doxorubicin (20).

Interestingly, a targeting peptide (iRGD) has been developed that combines the RGD motif with a protease site and a neuropilin targeting motif to create a peptide that promotes transendothelial passage of nanoparticles to enhance tissue penetration and targeting to tumor cells (21). The $\mathrm{F} 3$ peptide has also been used to deliver nanoparticles to the tumor microenvironment. A rat model of glioblastoma multiforme suggested that F3 targeted nanoparticles can be used for both tumor imaging and therapy (14).

Studies to date have all focused on in vitro studies or studies in rodents. One shortcoming of these studies is the lack of a demonstration of activity against human tumor vessels in vivo. A model of human tumor vasculature has recently been developed $(22,23)$. This model uses human embryonic stem cells as a source of vascular cells, thus the vascular cells are human. Vessels that are generated in this model demonstrate that they have both human endothelial and vascular smooth muscle cells.

We used the F3 peptide to deliver cisplatin loaded polyacrylamide (PAA) nanoparticles to tumor vessels in both murine and human ovarian cancer models. We demonstrate that these nanoparticles bind to murine and human tumor endothelial cells both in vitro and in vivo. When used as a therapeutic, these nanoparticles lead to significant tumor regression and then stabilization of tumor burden. Nanoparticles were effective in both xenograft and orthotopic tumor models. Our data strongly support the efficacy of vascular targeted nanoparticle therapy in ovarian cancer and represent the first demonstration of chemo-nanoparticle binding to human tumor vessels in vivo. 


\section{Materials and Methods \\ Nanoparticle (NP) Preparation}

Chemicals-Acrylamide, N,N,N',N'-tetraethylmethylenediamine (TEMED), Ammonium persulfate (APS), Polyethylene glycol dodecyl ether (Brij 30), 3-(acryloyloxy)-2hydroxypropylmethacrylamide (AHM), hexane, and dioctyl sulfosuccinate (AOT) were purchased from Sigma Aldrich. 3-(aminopropyl) methacrylamide (APMA) was obtained from Polysciences Inc.and ethanol (190 proof) was obtained from Fisher Scientific. Cisplatin was purchased from SICOR pharmaceuticals Inc. (Irvine, CA), while Sulfosuccinimidyl 4-[Nmaleimidomethyl] cyclohexane-1-carboxylate (Sulfo-SMCC), Traut's reagent (2-IT), amine reactive fluorescein isothiocyanate (FITC) and AlexaFluor 594 were from Thermo Scientific Inc. All solutions were prepared from $18 \mathrm{M}$ water purified by a Barnstead/Thermolyne Nanopure II system except where otherwise stated.

Blank Np polymerization-Hexane $(45 \mathrm{~mL})$ was added into a dried flask and stirred under a constant purge of argon. AOT (1.6 g) and Brij 30 (3.1 g) were added with continuous mixing under argon for $20 \mathrm{~min}$. Acrylamide $(0.711 \mathrm{~g})$ and APMA $(0.055 \mathrm{~g})$ were dissolved in PBS $(\mathrm{pH}=7.4)$ by sonication. AHM $(0.428 \mathrm{~g})$ was added to the acrylamide solution and the mixture was sonicated for 5 minutes to obtain uniformity. The acrylamide solution was then added to the hexane mixture and vigorously stirred for 20 minutes under argon protection.

Polymerization reaction was initiated with fresh ammonium persulfate (10\% aqueous solution, $40 \mu \mathrm{L})$ and TEMED $(40 \mu \mathrm{L})$ and vigorously mixing was continued for 12 hours. Hexane was then removed by rotary evaporation and nanoparticles were precipitated with ethanol. Surfactant and unreacted monomers were removed by washing with ethanol $(5 \times 160 \mathrm{~mL})$ followed by washing with water $(5 \times 100 \mathrm{~mL})$ in an Amicon ultra-filtration cell $(200 \mathrm{~mL}$, equipped with a Biomax $100 \mathrm{kDa}$ cutoff membrane). The concentrated $\mathrm{Np}$ were lyophilized for two days before use.

Cisplatin-Encapsulated and FITC or AlexaFluor linked Np-Cisplatin-encapsulated $\mathrm{Np}$ were polymerized as above except that $2 \mathrm{~mL}$ cisplatin (SICOR Pharnaceuticals Inc, Irvine, California, obtained via the Department of Pharmacy at the University of Michigan) drug solution $(1 \mathrm{mg} / \mathrm{mL})$ was used to dissolve all the monomers and Np were protected from light during the production process. An inductively coupled plasma (ICP) (Perkin-Elmer Optima 2000 DV with Winlab software) was used to determine the concentration of cisplatin encapsulated. The cisplatin concentration was measured to be $0.75 \pm 0.02 \mathrm{ug} / \mathrm{mg} \mathrm{Np}$ and nanoparticle solution was prepared to allow consistent dosing of cisplatin among experiments.

For fluorophore linked $\mathrm{Np}, 1 \mathrm{mg}$ of FITC or $5 \mathrm{mg}$ of AlexaFluor 594 was added into monomer solution and the mixture were kept stirring at $37^{\circ} \mathrm{C}$ for an hour before injection into hexane. Blank, fluorophore conjugated, or cisplatin encapsulated PAA Np, were prepared with a final average size of $24.4 \mathrm{~nm}$ (polydispersity index equals to 0.120 ).

F3 targeted Np-Cisplatin encapsulated, fluorophore linked, or blank Np were suspended in PBS by sonication, Sulfo-SMCC was added, solution was mixed for 60 minutes and then SMCC conjugated Np were washed by using Amicon centrifugal filter unit for 3 times. F3 peptide and 2-IT dissolved in DI water was then added into the SMCC conjugated Np suspension. The mixture was mixed overnight and then washed again to obtain the concentrated $\mathrm{Np}$.

Cell lines and in vitro Studies-ID8 and A2008 cells were a generous gift from Dr. George Coukos. SKOV3, HEY1, and A2780 cell lines were a generous gift of Dr. Rebecca Liu. Tumor endothelial cell (TEC) cultures were freshly generated from VE-Cadherin+/CD146+ cells 
FACS isolated from mechanically dissected tumors as previously described $(24,25)$. TEC were then grown in EBM2 media (Clonetech). Monocytes were isolated from $2 \mathrm{ml}$ of ACK lysed donor blood based upon their ability to adhere to plastic. All cells except TEC were grown in $\mathrm{RPMI} / 10 \% \mathrm{FBS} / 5 \%$ penicillin-streptomycin, medium and split two days prior to experiments to $40 \%$ confluency. For binding experiments, cells were incubated with a range of F3 targeted AlexaFluor 594 linked Np (F3-Alex-Np), or blank Np (10-100 $\mu \mathrm{g} / \mathrm{ml})$ for 4 hours with intermittent rocking. Cells were then washed $3 \mathrm{X}$ with PBS, incubated for 30 minutes and then imaged using a Nikon fluorescent microscope attached to CoolSNAP CCD camera (Roper Scientific). In order to determine in vitro killing efficiency cells were incubated with F3 targeted blank Np (F3-Np), F3 targeted cisplatin encapsulated Np (F3-Cis- $\mathrm{Np}, 0.15 \mu \mathrm{g} / \mathrm{ml}$ final cisplatin concentration), parental cisplatin compound (at $5 \mu \mathrm{g} / \mathrm{ml}$ final concentration for cell line experiments and at $1 \mathrm{ug} / \mathrm{ml}$ for TEC and PBMC experiment) or mock treated with PBS. The cells were washed after 4 hours and then allowed to grow for a total of 72 hours prior to harvesting for cell counting via trypan blue exclusion.

Mouse Studies-All mice were housed at the University of Michigan Medical School in the Unit for Laboratory Medicine (ULAM) and protocols were approved under the University Committee on the Use and Care of Animals (UCUCA). Tumor cell lines were grown in DMEM/ $10 \% \mathrm{FBS} / 5 \%$ penicillin-streptomycin, medium.

Axillary and flank tumor models: $10 \times 10^{6}$ cells were injected with $0.2 \mathrm{ml}$ of PBS and 300ul of matrigel (BD Biosciences). In the initial targeting experiments, axillary tumors were allowed to grow for 10 days and then $100 \mathrm{mg} / \mathrm{Kg} \mathrm{F} 3$ targeted Alexa-488 linked Np (F3-FITC-Np) or Alexa-488 linked Np (FITC-Np,) were administered intravenously. $24 \mathrm{hr}$ after injection the mice were sacrificed and tumors, liver, lung, kidney, heart and spleen were harvested and examined for fluorescent nanoparticle uptake. For therapeutic studies Hey1 tumor cells were stably transduced with a DsRed expressing lentivirus (plentiloxEV-DsRed virus, provided by the vector core at the University of Michigan). Tumor cells were injected subcutaneously into either the flank (ID8 studies) or axilla (SKOV3, A2780-GFP, and DsRED HEY1) of either C57B16 or nu/nu mice respectively. Axillary injection was used in the case of the human tumor xenografts as we find axillary tumors have a greater microvascular density than flank tumors. Xenografts were allowed to establish as indicated and were treated with either (1) IP cisplatin at $250 \mu \mathrm{g} / \mathrm{kg}$ alone or (2) IP cisplatin combined with with F3-Np via tail vein injection or (3) with F3-Cis-Np via tail vein injection (IV) at $100 \mathrm{mg} / \mathrm{kg}$ of nanoparticles (final cisplatin concentration $75 \mu \mathrm{g} / \mathrm{kg}$ ). Mice were treated initially at Day 10 and Day 14 (all tumor xenografts) and Day 21 (for ID8 and SKOV3 xenografts only). Tumor volume was monitored via caliper $\left(\mathrm{W}^{2} \times \mathrm{L} / 2\right)$. A2780-GFP mice were imaged with whole body imaging utilizing the Maestro imaging system. Mice were sacrificed on either day 18 or day 28. Axillary and flank tumors were harvested for histology and immunohistochemistry.

Intraperitoneal (IP) models: Mice were randomized by weight into treatment groups. 2.0 $\times 10^{6}$ ID8 cells harvested in exponential growth were injected intraperitoneally in $0.2 \mathrm{ml}$ of PBS. 10 days after tumor cell injection, control mice were then treated with either IP cisplatin at $250 \mu \mathrm{g} / \mathrm{kg}$ alone or along with (1) IP blank F3-Np, (2) IV blank F3-Np, or (3) both IP and IV administered blank F3-Np. Alternatively mice were treated with F3-Cis-NP (1) delivered IV, (2) IP, or (3) both IV and IP. Nanoparticles were dosed with final cisplatin does of 150 $\mu \mathrm{g} / \mathrm{kg} ; 150 \mu \mathrm{g} / \mathrm{kg}$ for IV or IP alone, or $75 \mu \mathrm{g} / \mathrm{kg}$ IV and $75 \mu \mathrm{g} / \mathrm{kg}$ IP for IV $/ \mathrm{IP}$ treated animals. Mice were treated at days 10,14, 21 and 28. Mice were followed for weight gain/ascites and sacrificed after a 10 gram weight gain or when they appeared moribund. Kaplan-Meier curves were plotted and statistical analysis performed via log rank test. 
Teratoma Model: Hey-1/teratomas were generated as previously described $(22,23)$. Briefly, H9 embryonic stem cells (ESC) were cultured on mouse embryonic fibroblasts, manually dispersed, and passaged. $1 \times 10^{6}$ undifferentiated $\mathrm{H} 9$ embryonic stem cells were injected subcutaneously into the axilla of NOD-SCID mice with matrigel and allowed to grow until teratomas were palpable. 100,000 DsRed-HEY1 cells were then injected within teratoma. Tumors were imaged using bio-immunofluorescence. Given the large size of the tumor/ teratomas and their rapid growth rates, mice were then treated with intravenous F3-Np or F3Cis-Np $75 \mu \mathrm{g} / \mathrm{kg}$ every 48 hour, 4 times, for a total of 8 days. Tumors were harvested 24 hours after the $4^{\text {th }} \mathrm{NP}$ treatment. Controls were treated with F3-FITC-Np 1 hour prior to sacrifice to confirm F3-NP targeting to human vessels. Tumors were then analyzed with Co-IF with antihuman CD31-PE.

\section{Results}

\section{Targeting of F3-targeted nanoparticles to tumor endothelial cells and tumor cells in vitro}

Blank, fluorophore conjugated or cisplatin encapsulated PAA Np, were prepared with a final average size ranging from $20-30 \mathrm{~nm}$ (Figure 1). The PAA nanoparticle is a hydrogel that has a high aqueous solubility and long plasma circulation time, being suitable for in vivo applications $(14,16,26)$. It also has excellent engineerability within both nanoparticle core and surface, which allowed conjugation of fluorophores and/or multiple targeting/visualization peptides for the current studies. F3 peptide was conjugated to the $\mathrm{Np}$ for targeting. This peptide, a subcomponent of the HMGN2 protein, has demonstrated specificity for both human tumor cells and tumor vasculature (13-15). Alexafluor-594 was bound to the nanoparticles (Alex$\mathrm{Np}$ ) for visualization in initial targeting experiments. To test the ability of the F3 peptide to target ovarian tumor cells, both mouse and human ovarian cancer lines (Mouse ID8 and Human SKOV3, A2008, A2780) were incubated with either F3-Alex-Np (100 $\mu \mathrm{g} / \mathrm{ml})$ or Alex-Np (1mg/ $\mathrm{ml}$ ). We observed significant binding of the $\mathrm{F} 3$ targeted $\mathrm{Np}$ to all ovarian tumor cell lines tested (Figure 2A). Little or no binding was observed with $\mathrm{Np}$ that lacked the targeting F3 peptide. We next tested the ability of F3-Np to bind to tumor endothelial cells (TECs) (Figure 2A). F3Alex-Np demonstrated strong binding to both mouse and human TECs. Once again, nontargeted Np showed little or no binding.

\section{In vitro cytotoxicity of F3 targeted cisplatin encapsulated Np}

In order to determine the cytotoxic potential of $\mathrm{F} 3$ targeted $\mathrm{Np}$, tumor cell lines were treated with either PBS, F3-Cis-Np, blank F3-Np combined with cisplatin, for four hours and then washed. The final concentration of cisplatin in the F3-Cis-Np was $0.15 \mu \mathrm{g} / \mathrm{ml}$ and that of cisplatin combined with the blank F3-Np was $1 \mu \mathrm{g} / \mathrm{ml}$ for TEC/PBMC experiments and $5 \mu \mathrm{g} /$ $\mathrm{ml}$ for A2780 and SKOV3 experiments. The cells were then allowed to grow for an additional 72 hours and harvested. We then assayed the number of viable cells in each treatment group relative to the PBS control. Consistent with previous studies of nanoparticles targeting tumor cells, our experiments demonstrated little cell death in the nanoparticle experimental groups (Figure 2B) (27-29).

We then repeated these experiments using tumor endothelial cells isolated from both murine ID8-VEGF ovarian tumors and human ovarian cancers. Unlike what was observed for the tumor cell lines, we observed significant induction of cell death with F3-Cis-Np on both mouse and human tumor endothelial cells. No cell death was noted when treating control PBMCs (Figure 2B). Thus F3 targeted Np appear to be more toxic to tumor endothelial cells than to tumor cell lines. 


\section{F3 -Np target tumor vessels in vivo}

To test the efficacy of F3 targeting in vivo we used the highly vascular ID8-VEGF ovarian tumors model (30). Mice bearing ID8-VEGF tumors were treated intravenously with increasing concentrations of either non-targeted Alexa488-Np or F3 targeted Alexa488 Np. Mice were sacrificed 24 hours after infusion and multiple tissue and tumor specimens were harvested and examined via fluorescence for nanoparticle localization. Highest specific binding to tumor vessels without significant uptake in liver and kidney was determined at a dose of $100 \mathrm{mg} / \mathrm{kg}$ (range tested $25-200 \mathrm{mg} / \mathrm{kg}$, Figure 2C and data not shown). At this dose, non-targeted FITC$\mathrm{Np}$ demonstrated little tumor specific uptake, but were found at significant levels in the liver and kidney. In contrast, at this concentration we observed significant uptake of F3-FITC-Np in tumor vessels and some uptake within tumor parenchyma. We noted minimal uptake of F3FITC-Np in kupffer cells of the liver and within the renal collecting tubules (Figure 2C and data not shown). No significant binding was observed in other tissues including the lung, heart, spleen, intestine, or brain (data not shown).

\section{Therapeutic efficacy of F3-Cis-Np}

Next, to test the therapeutic efficacy of the nanoparticles, ID8-VEGF tumors were grown in the axillas of mice for 14 days. Mice were then treated intravenously with two weekly doses of F3-Cis-NPs, with a final cisplatin concentration of $70 \mu \mathrm{g} / \mathrm{kg}$, IP cisplatin at $250 \mu \mathrm{g} / \mathrm{kg}$ or IP cisplatin at $250 \mu \mathrm{g} / \mathrm{kg}$, combined with intravenous blank F3-NPs (total $\mathrm{n}=15$ for cisplatin or cisplatin and F3-Np and $\mathrm{n}=20$ for F3-Cis-Np in two independent experiments) and then sacrificed one week later. Following the initial administration of F3-Cis-Np we observed a rapid and significant decline in tumor volume. This was maintained throughout the experiment. IP cisplatin alone and IP cisplatin/IV F3-NPs yielded identical results (data not shown). At the conclusion of the experiment, a 2.5 fold reduction in overall weight of the tumors and $\sim 3.5$ fold reduction in volume were noted when compared to IP cisplatin alone or IP cisplatin/IV F3-NPs (Figure 3A and data not shown). Histological analysis of these tumors demonstrated (1) large regions of hemorrhage and necrosis consistent with a potent anti-vascular effect and (2) a significant reduction in the size of tumor islets with an increase in stromal tissues (Figure 3B).

In order to determine the potential toxicity of this therapy, we collected serum from 3 animals in each treatment group 24 hours after the last intravenous treatment. Serum creatinine (a marker of renal function), aspartate aminotransferase and alanine aminotransferase (markers of hepatic function) and complete blood counts were not significantly different among untreated and F3-Cis-NP treated mice (Figure 3C). This suggests these Np are not excessively toxic to normal tissues. Further supporting the safety of the $\mathrm{Np}$, unlike mice treated with systemic cisplatin, we observed no significant weight loss for F3-Cis-Np treated mice nor any other adverse effects (data not shown).

We next tested the impact of $\mathrm{Np}$ on tumor growth using an orthotopic intraperitoneal tumor model. Tumors were allowed to engraft for 10 days and then mice were treated with either IP cisplatin at $250 \mu \mathrm{g} / \mathrm{kg}$ along and F3-Np IV, IP cisplatin alone, or F3-Cis-Np IV with a final concentration of $75 \mathrm{ug} / \mathrm{kg}$ cisplatin. We observed a significant increase in the overall survival of animals treated with the F3-Cis-Np as compared to IP cisplatin alone or along with F3-NPs (Figure 3D(1) and data not shown). To determine if combining IV F3-Cis-Np with IP F3-Cis$\mathrm{Np}$ therapy could improve survival further by targeting both tumor vasculature (via IV dosing) and tumor cells (via IP dosing), we repeated the orthotopic tumor studies and compared various combinations of Blank F3-Np and cisplatin delivered IV, IP or combined IP and IV versus F3Cis-Np via IV, IP, or IV and IP administration at $150 \mu \mathrm{g} / \mathrm{kg}$ (for IV or IP alone) or $75 \mu \mathrm{g} / \mathrm{kg}$ for the both IV and IP treatments for a total cisplatin concentration of $150 \mu \mathrm{g} / \mathrm{kg}$. Mice were treated on days 10, 14, 21 and 28 . We observed a significant survival advantage for all groups 
which received F3-Cis-Np intravenously as compared to mice receiving blank F3-Np combined with cisplatin. Interestingly, combined IV and IP treatment with F3-Cis-Np did not show a significant difference in survival (Figure 3D). This suggest that vascular exposure is the primary means of therapy as the addition of intraperitoneal therapy which could directly target tumor cells, had no added benefit.

We also tested the impact of F3-Cis-Nps using subcutaneous human tumor cell line xenograft tumor models. We used cisplatin sensitive Hey1 and A2780-GFP, and cisplatin resistant SKOV3 $\left(\mathrm{IC}_{50} 4 \mathrm{mM}\right)$ ovarian tumor cell lines ( $\mathrm{n}=11$ in treatment and control groups). As the human tumor xenografts with Hey1 and A2780 cells grow more rapidly than ID8 cells we used a more frequent treatment schedule, treating mice on Days 7, 10, and 14 after tumor engraftment with either F3-Cis-Np or blank Np and systemic cisplatin as described above. In the platinum sensitive Hey1 and A2780-GFP tumors there was clear arrest of tumor growth during the course of therapy as indicated both by tumor volumes and in vivo biofluorescent imaging (Figure 4A, $\mathrm{B}$ and data not shown). Consistent with an antivascular effect rather than an anti-tumor effect, we observed a similar growth arrest with therapy of the platinum resistant SKOV3 tumors. Tumors resected from these mice were extremely pale compared to controls (Figure 4B). Histochemical analysis of F3-Cis-Np treated tumors demonstrated a significant reduction in microvasculature and significant tumor necrosis similar to that observed with the ID8 tumors (data not shown).

\section{F3-Cis-Np target and eliminate human tumor vessels in vivo}

Finally, we wished to determine if the Np could bind to human tumor vessels in vivo. We used a recently developed model of human tumor vasculature which utilizes Hey1 tumors cells injected into H9 ESC derived teratomas. In this model tumor vascular cells are derived from the human ESC and thus are human in origin. We performed intravenous injections of F3Alexa-Np in H9-ESC-Hey1 tumor bearing mice. Importantly, we observed clear binding of the F3-Alexa-Np to the human $\mathrm{CD} 31^{+}$tumor vessels, confirming the ability of these F3 targeted $\mathrm{Np}$ to bind to human vessels in vivo. We therefore used this model to test the therapeutic efficacy of F3-Cis-Nps. To track tumor cell growth we used immunofluorescent DsRED Hey1 tumor cells. Once again we treated animals with either IV F3-Np and systemic cisplatin or F3-Cis$\mathrm{Np}(\mathrm{n}=3$ controls and $\mathrm{n}=3$ treatment groups in two independent experiments). Animals were treated on day 10,14, 17 and 21. Like the previous tumor models, treatment with F3-Cis-Np lead to an initial loss of fluorescence and then stabilization of disease, whereas tumors treated with systemic cisplatin demonstrated progressive growth (Figure 5).

\section{Discussion}

We used an F3-targeted polymeric nanoparticle formulation consisting of encapsulated cisplatin in a polyacrylamide nanoparticle to target tumor vessels as a cancer therapeutic. While vascular targeted nanoparticle studies have been reported previously, to our knowledge ours is the first to demonstrate the ability to bind to human tumor vessels in vivo.

In vitro studies demonstrated specific binding to tumor and tumor endothelial cells. In vivo studies demonstrated predominant binding to microvascular tumor endothelial cells with lesser uptake on tumor cells. Minimal non-specific uptake in kupffer cells of the liver, and renal excretion of nanoparticles were noted, however, non-specific binding was minimized with titration of dose. Minimal toxicity was noted with F3-Cis-Np treatment as evidenced by stable creatinine, liver function tests and complete blood counts. This is not surprising given the total cisplatin dose used in the nanoparticle studies ( 75 or $150 \mu \mathrm{g} / \mathrm{Kg}$ ) was $\sim 1 / 20^{\text {th }}$ the traditional dose of cisplatin $(1-5 \mathrm{mg} / \mathrm{kg})$. 
In addition to being safe, F3-targeted nanoparticles appear to be a highly effective as a therapeutic. While F3 peptides can bind both tumor cells and endothelial cells, our studies suggest that the primary effect of therapy was anti-vascular: Similar to prior studies, minimal in vitro cytotoxicity was noted with F3-Cis-Np treatment of human tumor cell lines (27-29). This may be due to the higher concentration of drug needed to kill tumor cells as evidenced by their higher IC50. In contrast we observed significant in vitro cytotoxicity of F3-Cis-Np on human tumor endothelial cells (Figure 2C). In addition we observed a rapid impact of therapy, associated with large regions of necrosis and hemorrhage, and a loss of clearly defined microvasculature consistent with an anti-vascular effect. Moreover, F3-Cis-Np were effective even in cisplatin resistant tumors. Finally, like other anti-angiogenics, continuous treatment was associated with stable disease and no further reduction of tumor was noted.

F3-Cis-Np therapy was effective in both solid tumor and IP tumor models. While the impact of therapy on flank tumors was most dramatic, a clear survival advantage was noted in the IP tumor model. The survival advantage was primarily associated with IV treatment. Interestingly, the combination of both IP and IV nanoparticles did not demonstrate a clear advantage over IV only nanoparticles. This may be because the cisplatin dose in the F3-Cis-Nps is subtherapeutic to kill tumor cells as observed in vivo. Alternatively, while we did not see significant systemic Np exposure with IP delivery (data not shown), it is possible the $\mathrm{Np}$ delivered IP are still primarily taken up in the tumor vasculature.

Based on previous observations that F3 peptide can target tumor cells, we chose to load our nanoparticles with cisplatinum, the most active anti-ovarian tumor cell agent. Given our observations that the F3 targeted nanoparticles appear to be primarily targeting tumor vascular cells, cisplatin may not be the most active chemotherapeutic. Cisplatin, a DNA targeting agent active against actively proliferating cells, may spare established non-dividing vascular cells. We hypothesize that F3-Np therapy with a microtubule targeting agent, may demonstrate even greater activity. Furthermore, an iRGD peptide was recently described (21). This peptide targets the vasculature and is then cleaved to allow release of the peptide with a now exposed neuropilin-1 binding motif which mediates penetration of the peptide through the vasculature to target tumor cells. Thus a combination of F3-Taxane nanoparticle and iRGD peptide targeted cisplatin nanoparticles could be particularly effective—targeting neo-vasculature, established tumor vessels, and tumor cells. Given the extremely low doses of chemotherapeutic agents necessary for effectiveness of the $\mathrm{Np}$ as demonstrated in the current study, such a combination could be possible with an acceptable side effect profile. Further experiments will be necessary to determine the appropriate sequencing for combining these agents; vascular disruption prior to the administration of anti-tumor agents could in theory reduce intratumoral drug delivery and thereby reduce efficacy. In this context, dynamic imaging studies may be useful to address the optimal scheduling of combinatorial therapy.

Finally, we show for the first time, the ability of vascular targeted Np to target human tumor endothelial cells, both in vitro and in vivo. We used a human ESC based tumor model in which a subset of the tumor vascular cells are derived from the human ESC and are therefore human tumor vessels (22). We have demonstrated that the vessels in this model are indeed tumor vessels, expressing unique tumor vascular markers (31). Thus we believe that this model represents an excellent tool for the study of therapeutics targeting human tumor vasculature.

In conclusion we have demonstrated that F3-targeted nanoparticles are a safe and potently effective therapeutic targeting human tumor endothelial cells. This proof of principle study demonstrates the ability to overcome ovarian cancer chemoresistance using vastly reduced drug concentrations by targeting nanoplatforms to the tumor neovasculature/ microenvironment. 


\section{Acknowledgments}

This work was initiated with the generous support of the Ovarian Cancer Research Fund Liz Tilberis' Award and the Damon Runyon Cancer Research Foundation Clinical Investigator Award. This work was completed with the support of the NIH New Investigator Innovator Directors Award grant \#00440377. We would like to thank the embryonic stem cell core at the University of Michigan for assistance with ESC culture.

\section{References}

1. Aghajanian C. The role of bevacizumab in ovarian cancer--an evolving story. Gynecol Oncol 2006;102 (2):131-3. [PubMed: 16846774]

2. Bidus MA, Webb JC, Seidman JD, et al. Sustained response to bevacizumab in refractory welldifferentiated ovarian neoplasms. Gynecologic oncology 2006;102(1):5-7. [PubMed: 16697451]

3. Monk BJ, Han E, Josephs-Cowan CA, et al. Salvage bevacizumab (rhuMAB VEGF)-based therapy after multiple prior cytotoxic regimens in advanced refractory epithelial ovarian cancer. [see comment]. Gynecologic oncology 2006;102(2):140-4. [PubMed: 16790264]

4. Numnum TM, Rocconi RP, Whitworth J, Barnes MN. The use of bevacizumab to palliate symptomatic ascites in patients with refractory ovarian carcinoma. Gynecol Oncol 2006;102(3):425-8. [PubMed: 16797681]

5. Garcia AA, Hirte H, Fleming G, et al. Phase II clinical trial of bevacizumab and low-dose metronomic oral cyclophosphamide in recurrent ovarian cancer: a trial of the California, Chicago, and Princess Margaret Hospital phase II consortia. J Clin Oncol 2008;26(1):76-82. [PubMed: 18165643]

6. Cohn DE, Valmadre S, Resnick KE, Eaton LA, Copeland LJ, Fowler JM. Bevacizumab and weekly taxane chemotherapy demonstrates activity in refractory ovarian cancer. Gynecol Oncol 2006;102(2): 134-9. [PubMed: 16527339]

7. Shojaei F, Wu X, Malik AK, et al. Tumor refractoriness to anti-VEGF treatment is mediated by CD11b +Gr1+ myeloid cells. Nat Biotechnol 2007;25(8):911-20. [PubMed: 17664940]

8. Buckanovich RJ, Sasaroli D, O’Brien-Jenkins A, et al. Tumor Vascular Proteins as Biomarkers in Ovarian Cancer. Journal of Clinical Oncology 2007;25 (7):852-61. [PubMed: 17327606]

9. Lu C, Bonome T, Li Y, et al. Gene alterations identified by expression profiling in tumor-associated endothelial cells from invasive ovarian carcinoma. Cancer Research 2007;67(4):1757-68. [PubMed: 17308118]

10. Temming K, Schiffelers RM, Molema G, Kok RJ. RGD-based strategies for selective delivery of therapeutics and imaging agents to the tumour vasculature. Drug Resistance Updates 2005;8(6):381402. [PubMed: 16309948]

11. Janssen ML, Oyen WJ, Dijkgraaf I, et al. Tumor targeting with radiolabeled alpha(v)beta(3) integrin binding peptides in a nude mouse model. Cancer Research 2002;62(21):6146-51. [PubMed: 12414640]

12. Garde SV, Forte AJ, Ge M, et al. Binding and internalization of NGR-peptide-targeted liposomal doxorubicin (TVT-DOX) in CD13-expressing cells and its antitumor effects. Anticancer Drugs 2007;18(10):1189-200. [PubMed: 17893520]

13. Christian S, Pilch J, Akerman ME, et al. Nucleolin expressed at the cell surface is a marker of endothelial cells in angiogenic blood vessels. Journal of Cell Biology 2003;163(4):871-8. [PubMed: 14638862]

14. Reddy GR, Bhojani MS, McConville P, et al. Vascular targeted nanoparticles for imaging and treatment of brain tumors. Clin Cancer Res 2006;12(22):6677-86. [PubMed: 17121886]

15. Porkka K, Laakkonen P, Hoffman JA, Bernasconi M, Ruoslahti E. A fragment of the HMGN2 protein homes to the nuclei of tumor cells and tumor endothelial cells in vivo. Proceedings of the National Academy of Sciences of the United States of America 2002;99(11):7444-9. [PubMed: 12032302]

16. Koo YE, Reddy GR, Bhojani M, et al. Brain cancer diagnosis and therapy with nanoplatforms. Adv Drug Deliv Rev 2006;58(14):1556-77. [PubMed: 17107738]

17. Kopelman R, Koo YEL, Philbert M, et al. Multifunctional nanoparticle platforms for in vivo MRI enhancement and photodynamic therapy of a rat brain cancer. J Magn Magn Mater 2005;293:40410 . 
18. Mukhopadhyay S, Barnes CM, Haskel A, Short SM, Barnes KR, Lippard SJ. Conjugated platinum (IV)-peptide complexes for targeting angiogenic tumor vasculature. Bioconjug Chem 2008;19(1): 39-49. [PubMed: 17845003]

19. Lu Z, Tsai M, Lu D, et al. Tumor-penetrating microparticles for intraperitoneal therapy of ovarian cancer. Journal of Pharmacology \& Experimental Therapeutics 2008;327(3):673-82. [PubMed: 18780831]

20. Pastorino F, Di Paolo D, Piccardi F, et al. Enhanced antitumor efficacy of clinical-grade vasculaturetargeted liposomal doxorubicin. Clinical Cancer Research 2008;14(22):7320-9. [PubMed: 19010847]

21. Sugahara KN, Teesalu T, Karmali PP, et al. Tissue-penetrating delivery of compounds and nanoparticles into tumors. Cancer Cell 2009;16(6):510-20. [PubMed: 19962669]

22. Tzukerman M, Rosenberg T, Reiter I, et al. The influence of a human embryonic stem cell-derived microenvironment on targeting of human solid tumor xenografts. Cancer Res 2006;66(7):3792-801. [PubMed: 16585206]

23. Tzukerman M, Rosenberg T, Ravel Y, Reiter I, Coleman R, Skorecki K. An experimental platform for studying growth and invasiveness of tumor cells within teratomas derived from human embryonic stem cells. Proc Natl Acad Sci U S A 2003;100(23):13507-12. [PubMed: 14573705]

24. Pulaski HL, Spahlinger G, Silva IA, et al. Identifying alemtuzumab as an anti-myeloid cell antiangiogenic therapy for the treatment of ovarian cancer. Journal of Translational Medicine 2009;7:49. [PubMed: 19545375]

25. Conejo-Garcia JR, Buckanovich RJ, Benencia F, et al. Vascular leukocytes contribute to tumor vascularization. Blood 2005;105(2):679-81. [PubMed: 15358628]

26. Koo YE, Fan W, Hah H, et al. Photonic explorers based on multifunctional nanoplatforms for biosensing and photodynamic therapy. Appl Opt 2007;46(10):1924-30. [PubMed: 17356639]

27. Jin W, Xu P, Zhan Y, et al. Degradable cisplatin-releasing core-shell nanogels from zwitterionic poly (beta -aminoester)-graft-PEG for cancer chemotherapy. Drug Deliv 2007;14(5):279-86. [PubMed: 17613015]

28. Uchino H, Matsumura Y, Negishi T, et al. Cisplatin-incorporating polymeric micelles (NC-6004) can reduce nephrotoxicity and neurotoxicity of cisplatin in rats. Br J Cancer 2005;93(6):678-87. [PubMed: 16222314]

29. Nishiyama N, Okazaki S, Cabral H, et al. Novel cisplatin-incorporated polymeric micelles can eradicate solid tumors in mice. Cancer Res 2003;63(24):8977-83. [PubMed: 14695216]

30. Zhang L, Yang N, Garcia JR, et al. Generation of a syngeneic mouse model to study the effects of vascular endothelial growth factor in ovarian carcinoma. American Journal of Pathology 2002;161 (6):2295-309. [PubMed: 12466143]

31. McLean K, Pulaski HS, Wang Y, Spahlinger G, Nor JE, RJB. Development of a Mouse Ovarian Cancer Model with Human Tumor Vessels to Test Novel Anti-Vascular Therapeutics. Gynecologic oncology 2009;112(2):S10-S1. 

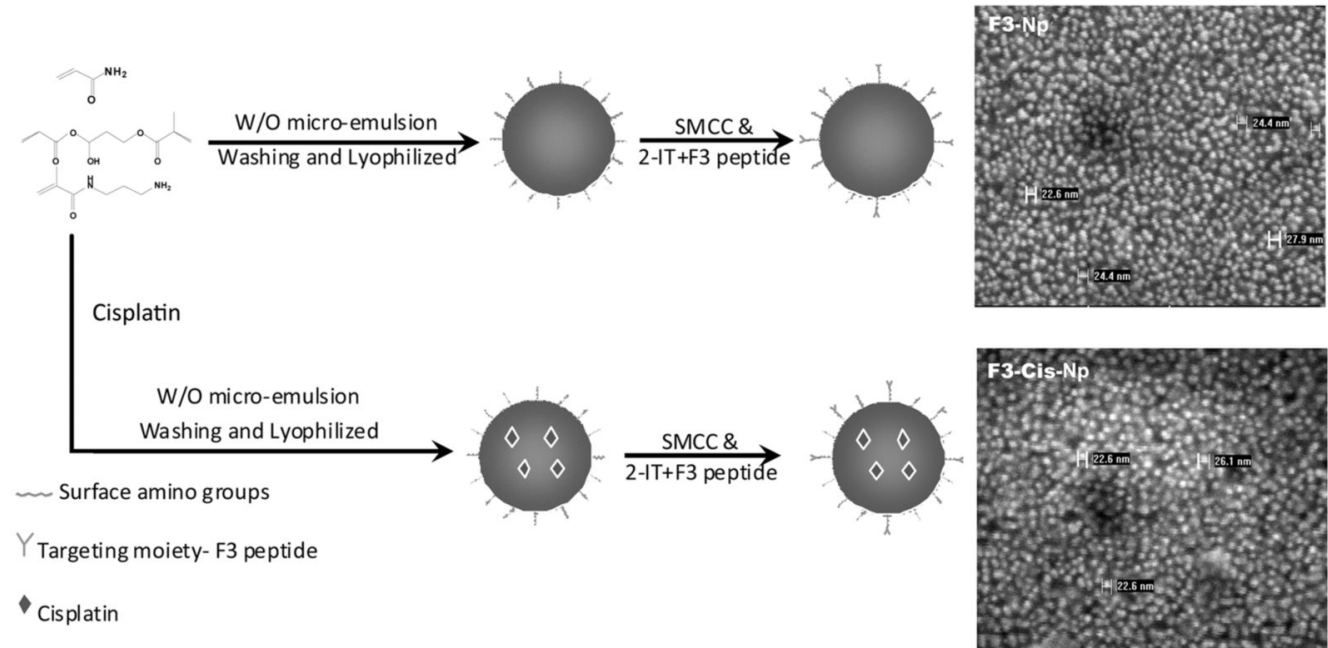

Figure 1. Development of nanoparticles

A Schematic demonstration of the production of both blank and cisplatin containing F3 targeted nanoparticles. B. The scanning electron micrographs showing the size of the nanoparticle after modification. 

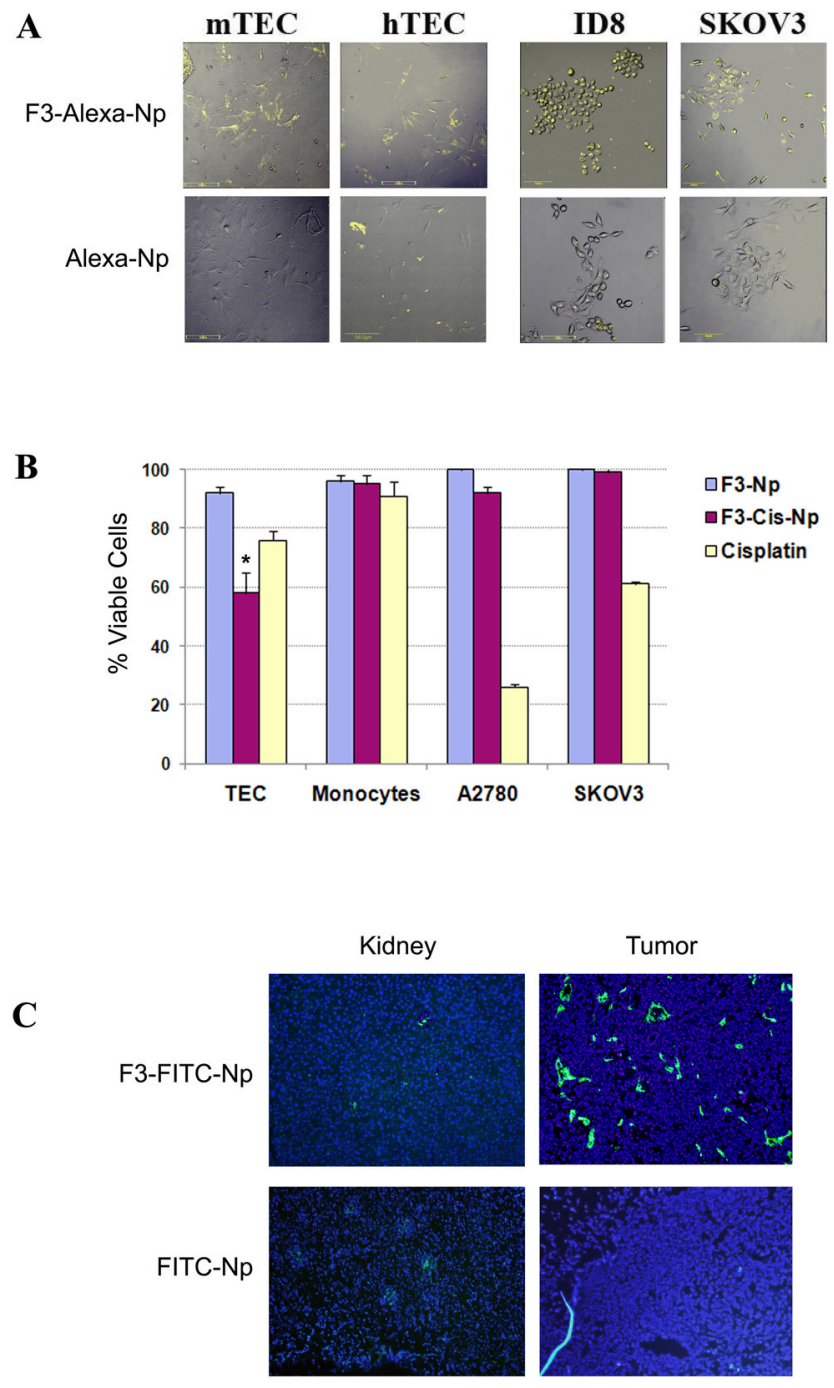

Figure 2. Binding and cytotoxicity of F3 targeted nanoparticles

A. In vitro binding of F3 targeted Alexa-594 nanoparticles (F3-Alexa-Np) but not for nontargeted Alexa-594 control nanoparticles (Alexa-Np) to murine and human tumor endothelial cells (mTEC and hTEC) and murine (ID8) and human (SKOV3) tumor cell lines. B. Percent viable cells (relative to PBS controls) following treatment with F3-Cis-Np, blank F3-Np, or cisplatin ( $1 \mu \mathrm{g} / \mathrm{ml}$ for TECs and $5 \mu \mathrm{g} / \mathrm{ml}$ for tumor cells) targeting human tumor endothelial cells (TEC), human peripheral blood monocytes, and ovarian tumor cells (SKOV3 and A2780). C. In vivo binding of F3-Alexa488- Np and non-targeted control Alexa-488 Np in murine ID8 flank tumors and kidneys of treated mice. 
A.

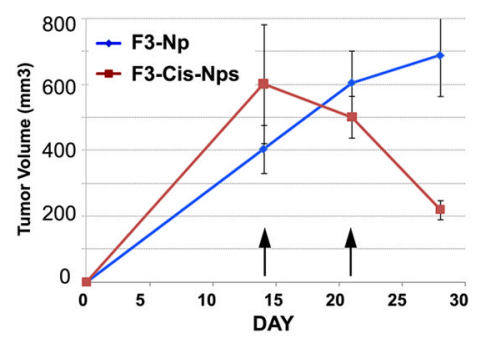

B.

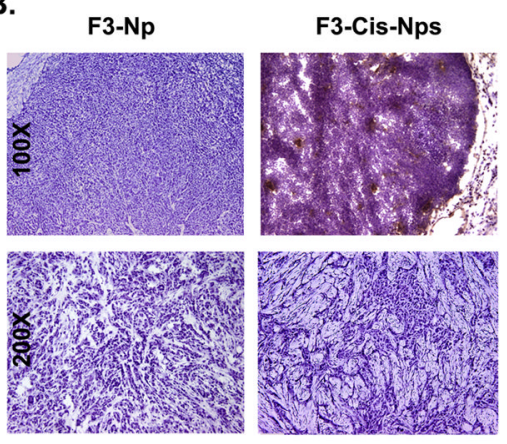

D.(1)

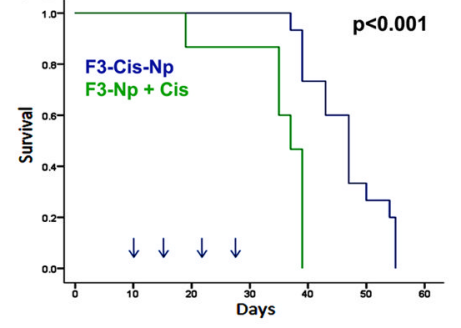

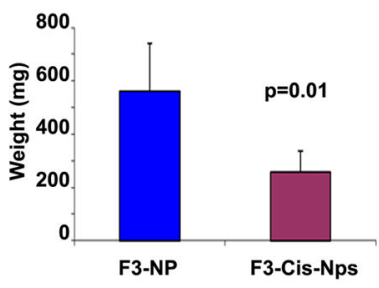

C.

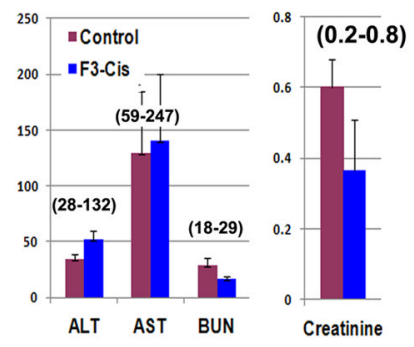

(2)

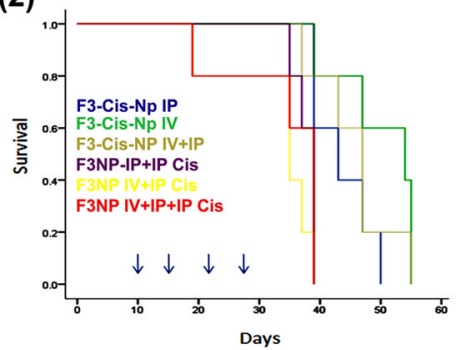

Figure 3. Therapeutic efficacy and toxicity of F3-Cisplatin-nanoparticles in a murine ovarian tumor model

A. Tumor growth curves and weights of ID8 xenografts treated with F3-Cis-Np or blank F3$\mathrm{Np}$ and systemic cisplatin ( $\mathrm{n}=15$ animals per group). Arrows indicate time of treatment. B. Tissue histology of tumors from blank F3-Np and systemic cisplatin (F3-Np + Cis) or F3-Cis$\mathrm{Np}$ treated tumors. Low power image demonstrates large area of vascular necrosis (upper right) in treated tumors. High power images demonstrate significant reductions in tumor islets in treated tumors (lower right). C. Lack of toxicity in F3-Cis-Np treated animals as demonstrated alanine aminotranferease (ALT) and aspartate aminotransferase (AST), blood urea nitrogen (BUN) and serum creatinine from mice treated with F3-Cis-Nps or F3-Np controls. Normal ranges are indicated (parentheses). D. Kaplan Meier curves indicating survival using an orthotopic intraperitoneal tumor model (1) comparing intravenous (IV) F3-Cis-Np vs IV control nanoparticles + systemic cisplatin delivered intraperitoneally (F3-Np + Cis), and (2) comparison of indicated treatment groups comparing IV and IP treatment of F3-Cis-Np and various controls. Greatest survival advantage was seen in the IV F3-Cis-Np group. Addition of IP F3-Cis-Np added no survival advantage ( $\mathrm{n}=10$ animals per group). Arrows indicate time of treatment. 
$A(1)$

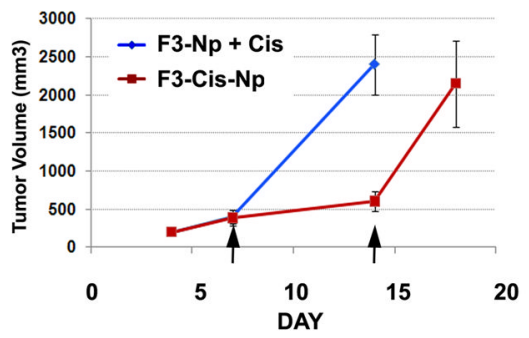

B (1)

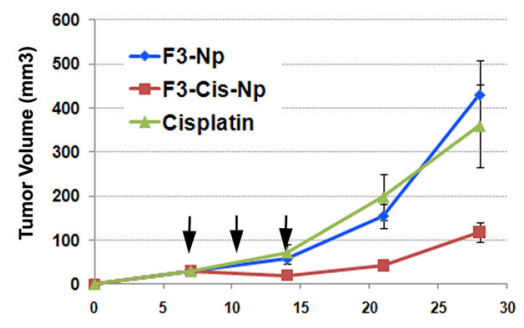

(2)

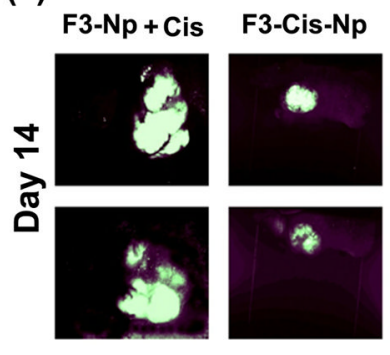

(2)

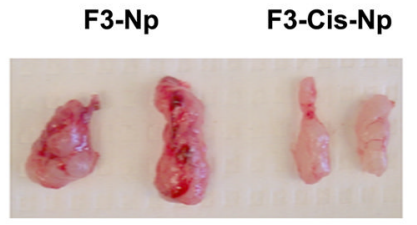

Figure 4. Therapeutic efficacy of F3-Cis-Np against human tumor xenografts

A. (1)Tumor growth curves and (2) in vivo fluorescent imaging of F3-NP + cisplatin or F3Cis-Np treated A2780 cisplatin sensitive tumor xenografts. B (1) and (2). Tumor growth curves and gross tumor pathology of F3-NP + cisplatin or F3-Cis-Np treated cisplatin resistant SKOV3 tumor xenografts. F3-Cis-Np treated tumors were significantly smaller and pale/avascular ( $\mathrm{n}=5$ /group in independent experiments). 
A
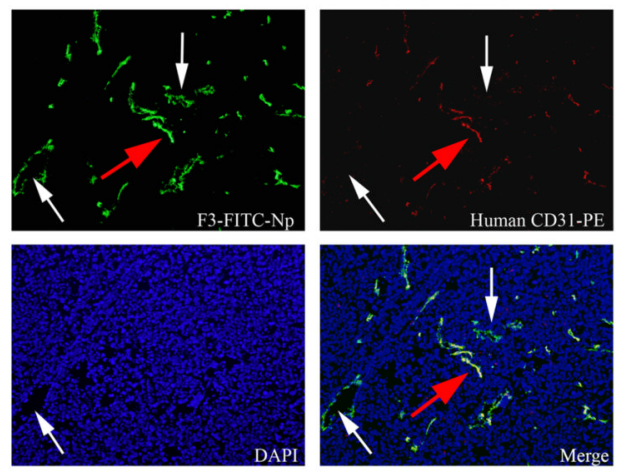

B

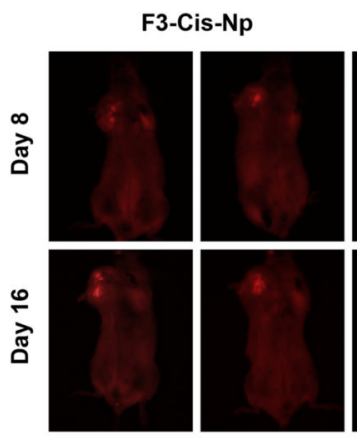

Control Np

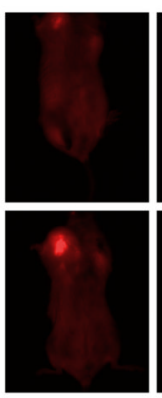

C

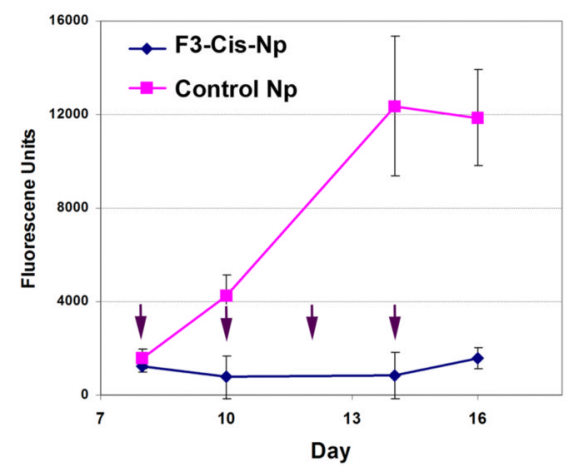

D

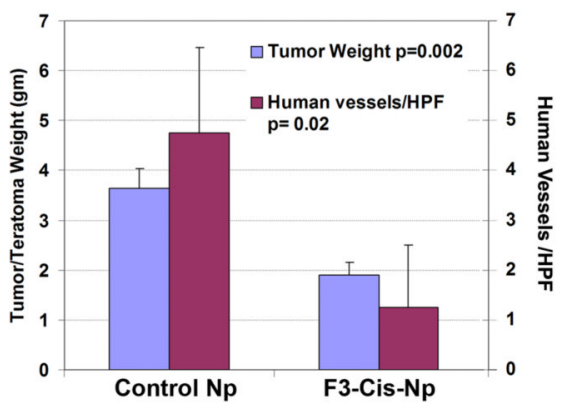

Figure 5. F3 targeted Np effectively target human tumor vessel $\mathrm{s}$ in vivo

A. Immunofluorescence demonstrating the F3-Alexa488-Np (F3-Alexa-Np) bind to human $\mathrm{CD} 31+(\mathrm{hCD} 31)$ vessels in vivo using a human embryonic stem cell tumor model with human vessels. Red arrow indicates hCD31+ human vessel. White arrows identify hCD31(-) murine vessels. B. In vivo fluorescent imaging of F3-Cis-Np and blank F3-Np treated ESC-DsREd Hey1 tumors just prior to the first IV treatments (day 8) and at the time of sacrifice two days after the last IV treatment (day 16). C. Tumor growth curves of F3-Cis-Np treated and control (F3-Np) treated ESC-DsREd Hey1 tumors. Arrows indicate IV NP treatment ( $\mathrm{n}=3$ per group in two independent experiments). D. Tumor weight and density of human vessels in F3-CisNp treated and F3-Np treated ESC-DsREd Hey1 tumors. Both were statistically significantly reduced as indicated. 